square wave. For odd half-cycles of the modulating wave the value of the current corresponds to the lower limit of a size-range, and for even half-cycles to the upper limit of the range. The images of particles within the size-range can be distinguished from others in the field of view by their characteristic feature of overlapping during odd half-cycles and not overlapping during even half-cycles. The images of particles smaller than the lower limit do not overlap during either half-cycle, while the images of particles larger than the upper limit overlap all the time. A switch enables each size-range with a corresponding counter to be selected in turn. Each time a particle with the characteristic feature mentioned above is seen, a triggering pulse (initiated by a foot switch) is routed to the counter appropriate to the size-range.

Medical Research Council

V. TIMBRELI

Pneumokoniosis Research Unit (S. Wales),

Llandough Hospital

Nr. Cardiff.

May 29.

\section{Interaction of Macromolecules and Fibrinogen}

RICKETTS ${ }^{1}$ has described an interaction between dextran and fibrinogen. It was therefore decided to investigate the interaction of several other polymers, used in transfusion therapy, with fibrinogen of human and bovine origin.

Dextran fractions of intrinsic viscosities $0.41,0.31$, 0.24 and 0.06 , and possessing branching factors (ratios of $\alpha$ 1-6 glucosidic linkage to $\alpha$ 1-4 glucosidic linkage) varying from $4: 1$ to $11: 1$ were used. A commercial preparation of polyvinylpyrrolidone, gum acacia in saline, and a hydrolysed laminarin fraction of intrinsic viscosity 0.2 , were also investigated.

Solutions containing $1 \cdot 5,3.0$ and 6.0 per cent of the polymers in 0.9 per cent saline were each added to equal volumes of solutions containing $0.4,0.8$ and 1.5 per cent of fibrinogen also in 0.9 per cent saline. The tests were made under the following conditions.

(1) Solutions mixed at $25^{\circ} \mathrm{C}$. and then placed in a refrigerator at $4^{\circ} \mathrm{C}$. for $16 \mathrm{hr}$. All the dextran fractions, with the exception of the one of intrinsic viscosity 0.06, gave a precipitate. Polyvinylpyrrolidone, laminarin and gum saline gave precipitates of similar magnitude to the dextran fractions of intrinsic viscosity 0.41 and 0.24 . The amount of precipitate was greater with increasing concentrations of both fibrinogen and polymer.

(2) Solutions mixed at $37^{\circ} \mathrm{C}$. and then placed in an incubator at $37^{\circ} \mathrm{C}$. for $16 \mathrm{hr}$. Only laminarin formed an insoluble complex at this temperature; but when the temperature of the remaining solutions was lowered to $4^{\circ} \mathrm{C}$., precipitation of the complex took place with each of the other polymers.

Dilutions of the polymers at concentrations 0.75 , 1.5 and 3 per cent were made at $37^{\circ} \mathrm{C}$. in plasma. An aliquot was incubated at $37^{\circ} \mathrm{C}$. during $8 \mathrm{hr}$. and another at $4^{\circ} \mathrm{C}$. during a similar period. No precipitation occurred with any polymer at either temperature. However, complex formation did take place in a 5 per cent albumin solution containing 1 per cent sodium citrate, 0.9 per cent saline and 0.4 per cent fibrinogen, the reaction being carried out as in method (1). This indicates that it is not just simple solubility in citrate or albumin which is preventing the formation of the complex. The dextran-fibrinogen complex was prepared on a larger scale and injected intravenously into mice at a doselevel of $300 \mathrm{mgm}$. per $\mathrm{kgm}$. of body-weight. None of the mice showed any untoward reaction.

It is well known that dextran fractions above a certain critical molecular size, namely, 59,000, increase the sedimentation-rate of erythrocytes ${ }^{2}$. Solutions containing 1 and 2 per cent of dextran (intrinsic viscosity 0.31 ) were made in a 5 per cent albumin solution. Red blood cells, washed free from plasma with saline, were added to this solution; the erythrocyte sedimentation-rate in the albumindextran solution was of the same order of magnitude as that of blood containing a similar dextran concentration. Hence the removal of fibrinogen had not affected the sedimentation-rate of erythrocytes. The electrophoretic pattern of plasma containing dextran is being examined to see if it is possible to detect any evidence of a soluble dextran-fibrinogen complex.

These preliminary investigations indicate that the property of forming a complex with fibrinogen is not peculiar to dextran but is also possessed by polyvinylpyrrolidone, gum acacia and laminarin, and since the complex formed contains only approximately 1 per cent of the polymer in solution and 10 per cent of fibrinogen, it probably would have no pathological significance.

$$
\begin{aligned}
& \text { F. Fletrcher } \\
& \text { L. E. Martin }
\end{aligned}
$$

Benger Research Laboratories, Holmes Chapel, Cheshire.

Department of Surgery, University, Manchester. July 3.

${ }^{2}$ Ricketts, C. R., Nature, 169, 970 (1952).

${ }^{2}$ Thorsén, G., Acta Chir. Scand., supp. 154 (1950).

\section{Occultation of a Radio Star by the Solar Corona}

IT has been suggested ${ }^{1}$ that the radiation from a radio star might be cut off by refraction in the solar corona, even when the angular separation of the sun and the star is several times the angular radius of the visible disk $\left(R_{\odot}\right)$. The existing data on the coronal electron density have been used for predicting the effective radius of the sun for occultation on various frequencies ${ }^{2}$. Link ${ }^{2}$ has calculated the circumstances of the occultation for 1952 and has also shown that the observed intensity of the radiation from the radio star should increase before the actual occultation. Attempts were made to observe these effects in June 1950 and 1951 when the radio star in Taurus $(05.01)$ passed within an angular distance of about $4.5 R_{\odot}$ from the sun's centre, but intense sunspot radiation prevented any useful results being obtained.

Measurements of the intensity of the radio star 05.01 were again made on frequencies of $210,81.5$ and $38 \mathrm{Mc} / \mathrm{s}$. during the period June 5-20, 1952 ; as in the earlier observations, interferometers of large resolving power. were used for reducing the amplitude of the record from the undisturbed sun. In addition, the position of the star was measured each day on $81.5 \mathrm{Mc} . / \mathrm{s}$.

No accurate observations were possible on 210 Mc./s., owing to the disturbing effect of a small sunspot ; but reliable results were obtained on both 81.5 